\title{
Quantum dynamics of a superconducting tunnel junction
}

\author{
Ulrich Eckern \\ Institut für Theorie der Kondensierten Materie, Universität Karlsruhe, D-7500 Karlsruhe 1, Federal Republic of Germany \\ Gerd Schön* and Vinay Ambegaokar ${ }^{\dagger}$ \\ Institute for Theoretical Physics, University of California, Santa Barbara, California 93106
}

(Received 18 June 1984)

\begin{abstract}
Basing our model and method on the microscopic theory we formulate a quantum-mechanical description for the relevant variable in a superconducting tunnel junction, i.e., the phase difference across the junction. The quasiparticle degrees of freedom are responsible for dissipation and noise in the system. Because of the discreteness of the charge-transfer process, the noise is shot noise. The energy gaps in the superconductors lead to further interesting features. We discuss the consequences of these physical effects on macroscopic quantum phenomena.
\end{abstract}

\section{INTRODUCTION}

The dynamical variable that describes a Josephson junction is the phase difference of the order parameters of the superconductors on the two sides of the barrier. It has been known for some time that this variable is coupled to microscopic degrees of freedom, the quasiparticle excitations, which act as a heat reservoir and source of phase fluctuations. Various schemes have been put forward over the years to describe this coupling. The most physically appealing of these ideas is the so-called resistively shunted junction model. ${ }^{1,2}$ In its usual form this model treats the phase as a classical variable, and the barrier as a linear resistor with its associated classical thermal noise. To describe interesting physical situations that occur at very low temperatures, generalizations of this simple model are needed: A proper quantum-mechanical treatment of the dynamics of the phase and the environment of quasiparticles is required; and, one might wish to take into account the fact that junctions by themselves, i.e., without shunt resistors across them, are typically not linear resistances but display the nonlinearities associated with the energy gaps in the superconductors. A suitably general formalism, based on a microscopic model and satisfying these requirements, has been briefly described in a note by the present authors., When the nonlinear conductance is ignored and the phase difference is assumed to be small the results of this model have been shown to be equivalent to those obtained from Caldeira's and Leggett's ${ }^{5,6}$ model treatment of linear (Ohmic) dissipation.

In this paper we describe our model and method in more detail. In addition to making connection with the case of linear dissipation, which was the useful thing to do at the time of our original publication, we emphasize the interesting aspects of our nonlinear model. We work out the consequences of the theory for switching by quantum-mechanical tunneling from a superconducting to a resistive state, we examine the question of coherent oscillations in a bistable potential well, and we display the novel Langevin equation our model leads to when the condition of least action is imposed.
In outline, the plan of this paper is as follows. In the next section we introduce the microscopic model, and show how to express its statistical properties as a functional integral over the relevant slow variable, the phase. We show how bulk energies suppress fluctuations in the magnitudes of the order parameters in the superconductors on the two sides of the barrier and also couple the time derivative of the phase to the voltage difference across the junction. These points were contained in Ref. 3. We go beyond that work by including the effect of a magnetic field and deriving the equations for statistical fluctuations in a ring containing an oxide barrier. We also show that for phase variations slow on the scale $\hbar / \Delta$ where $\Delta$ is the energy gap, the dominant effect of the quasiparticle environment at zero temperature is to renormalize the capacitance of the junction. In Sec. III we discuss the time evolution of the reduced density matrix (i.e., traced over all degrees of freedom except the phase) and obtain a functional integral form similar to that given by Feynman and Vernon, ${ }^{7}$ Caldeira and Leggett, ${ }^{6}$ and Schmid. ${ }^{8}$ Our formula contains the fluctuation and dissipation response functions of the system, and thus goes beyond the linear dissipation treated in Refs. 5, 6, and 8 . In Sec. IV we show that in the classical limit the time evolution is determined by a Langevin equation in which the autocorrelation function of the noise current depends on the time-dependent phase, and that this corresponds to shot noise. Finally, we discuss the consequences of the physical effects we have included on macroscopic quantum effects in tunnel junctions.

\section{QUANTUM STATISTICS OF A SUPERCONDUCTING TUNNEL JUNCTION}

A particular virtue of superconductors and superconducting tunnel junctions is their property that they can be described by a fairly simple microscopic model, which has been shown to cover most of the essential physics. As far as the bulk superconductor is concerned the description can be based on the BCS Hamiltonian, ${ }^{9}$ which assumes an effective local, attractive interaction 


$$
\begin{aligned}
H= & \int d^{3} x \psi_{\sigma}^{\dagger}(x) \frac{-\hbar^{2}}{2 m}\left(\vec{\nabla}-\frac{i e}{\hbar c} \overrightarrow{\mathrm{A}}\right)^{2} \psi_{\sigma}(x) \\
& -\frac{g}{2} \int d^{3} x \psi_{\sigma}^{\dagger}(x) \psi_{-\sigma}^{\dagger}(x) \psi_{-\sigma}(x) \psi_{\sigma}(x) \\
& +\int d^{3} x \frac{1}{8 \pi}\left(h-h_{\mathrm{ext}}\right)^{2}
\end{aligned}
$$

(The notations are standard, a summation over spins is implied, and $h_{\text {ext }}$ denotes an externally applied magnetic field.) A superconducting tunnel junction consists of two such superconductors ( $L$ and $R$ for left and right) which are coupled. It is described by

$$
H=H_{L}+H_{R}+H_{T}+H_{Q},
$$

where, e.g., $H_{L}\left(\psi_{L \sigma}\right)$ is of the form (1), with $\psi_{L \sigma}(x)$ referring to electrons in the left electrode. The coupling is due to the transfer of electrons between $L$ and $R$ and due to Coulomb interaction across the barrier. The former can be described by the tunneling Hamiltonian ${ }^{10}$

$H_{T}=\int_{x \in L} d^{3} x \int_{x \in R} d^{3} x^{\prime}\left[T_{x x^{\prime}} \psi_{L \sigma}^{\dagger}(x) \psi_{R \sigma}\left(x^{\prime}\right)+\right.$ H.c. $]$.

The range of the tunneling matrix element $T_{x x^{\prime}}$ is restricted to the vicinity of the oxide barrier. This simple form of $H_{T}$ has proven to be very successful. As long as we are concerned only with time scales slow compared with the inverse plasma frequency, the Coulomb interaction becomes an effective capacitive interaction depending only on the total charges on each electrode

$$
H_{Q}=\frac{1}{8 C}\left(Q_{L}-Q_{R}\right)^{2}
$$

Here $C$ is the usual capacitance determined by the geometry and properties of the insulator. The charge on the left side is

$$
Q_{L}=e \int d^{3} x \psi_{L \sigma}^{\dagger}(x) \psi_{L \sigma}(x)
$$

A superconducting ring with a weak link, i.e., a rf superconducting quantum-interference device (SQUID), in cases where this weak link is a tunnel barrier, can also be described by a Hamiltonian of the form (2) (with $L$ and $R$ joined in a loop).
Quantum-statistical properties of the system can be obtained from the grand canonical partition function

$$
Z_{G}=\operatorname{Tr}_{\psi, \vec{A}}\{\exp [-\beta(H-\mu N)]\},
$$

or, more generally, from a generating functional $Z(\xi)$ where $\xi$ couples to the quantity of interest. The trace is taken over the fermion fields $\psi$ and the vector potential $\overrightarrow{\mathrm{A}}$. We now eliminate the quartic interactions in $H$ by means of the Hubbard-Stratonovich procedure. ${ }^{11}$. In the problem of a superconducting tunnel junction this is achieved by introducing complex order-parameter fields $\Delta_{L}(x \tau)$ and $\Delta_{R}(x \tau)$ coupling to $\psi_{L \uparrow}^{\dagger}(x) \psi_{L \downarrow}^{\dagger}(x)$ in the $L$ electrode, and similar in $R$. They account for the attractive BCS interactions. Furthermore, we need a real voltage field $V(\tau)$, coupling to the charges, to account for the Coulomb interaction. The resulting effective Hamiltonian is a bilinear form in the four-dimensional space of the spinors $\Psi$ and $\Psi^{\dagger}=\left(\psi_{L \uparrow}^{\dagger}, \psi_{L \downarrow}, \psi_{R \uparrow}^{\dagger}, \psi_{R \downarrow}\right)$. As a result the trace over the fermion fields can be explicitly performed and we arrive at a path-integral representation (in imaginary times) of the partition function ${ }^{12}$

$$
\begin{aligned}
Z_{G}=\int & D^{2} \Delta_{L}(x \tau) D^{2} \Delta_{R}(x \tau) D V(\tau) D \overrightarrow{\mathrm{A}}(x \tau) \\
& \times e^{-S\left[\Delta_{L}, \Delta_{R}, V, \overrightarrow{\mathrm{A}}\right] / \hbar},
\end{aligned}
$$

where the action is

$$
\begin{aligned}
-S / \hbar= & \operatorname{Tr} \ln \overline{\widehat{G}}-1-\int_{0}^{\hbar \beta} \frac{d \tau}{\hbar} \int_{x \in L} d^{3} x \frac{1}{g_{L}}\left|\Delta_{L}(x \tau)\right|^{2} \\
& -(R \leftrightarrow L)-\int_{0}^{\hbar \beta} \frac{d \tau}{\hbar} \frac{1}{2} C V^{2}(\tau) \\
& -\int_{0}^{\hbar \beta} \frac{d \tau}{\hbar} \int d^{3} x \frac{1}{8 \pi}\left[\left(h(x \tau)-h_{\mathrm{ext}}\right]^{2}\right.
\end{aligned}
$$

Here $\overline{\widehat{G}}$ is a $4 \times 4$ matrix Green's function in the space spanned by $L$ and $R$ and in the particle-hole space ${ }^{13}$ characteristic of superconductivity. It depends on the fields $\Delta_{L}, \Delta_{R}$, and $V$ and its inverse is given by

$$
\overline{\widehat{G}}^{-1}\left(x \tau, x^{\prime} \tau^{\prime}\right)=\left(\begin{array}{cc}
\hat{G}_{L}^{-1}\left(x \tau, x^{\prime} \tau^{\prime}\right) & -\widehat{T}_{x x^{\prime}} \delta\left(\tau-\tau^{\prime}\right) \\
-\hat{T}_{x x^{\prime}}^{\dagger} \delta\left(\tau-\tau^{\prime}\right) & \widehat{G}_{R}^{-1}\left(x \tau, x^{\prime} \tau^{\prime}\right)
\end{array}\right) .
$$

The diagonal elements in the $L-R$ space are

$$
\widehat{G}_{L(R)}^{-1}\left(x \tau, x^{\prime} \tau^{\prime}\right)=\left\{-\hbar \frac{\partial}{\partial \tau} \hat{\imath}+\left[\frac{\hbar^{2}}{2 m}\left[\vec{\nabla}-\frac{i e}{\hbar c} \overrightarrow{\mathrm{A}} \widehat{\tau}_{3}\right]^{2}+\mu(\bar{\mp}) \frac{i e}{2} V(\tau)\right] \widehat{\tau}_{3}-\widehat{\Delta}_{L(R)}(x \tau)\right\} \delta\left(x-x^{\prime}\right) \delta\left(\tau-\tau^{\prime}\right)
$$

where $\widehat{\tau}_{3}$ is the Pauli matrix in the particle-hole space. The order-parameter field appears off-diagonal in this space,

$$
\widehat{\Delta}(x \tau)=\left[\begin{array}{cc}
0 & \Delta(x \tau) \\
\Delta^{*}(x \tau) & 0
\end{array}\right]=|\Delta| e^{-i \phi \hat{\tau}_{3}} \widehat{\tau}_{1} .
$$

The two electrodes $L$ and $R$ are coupled by

$$
\widehat{T}_{x x^{\prime}}=\left(\begin{array}{cc}
T_{x x^{\prime}} & 0 \\
0 & -T_{x x^{\prime}}^{*}
\end{array}\right)
$$

At this point it is advantageous to perform a gauge transformation which makes the particle-hole offdiagonal elements real and explicitly displays the role of the phases $\phi_{L(R)}(x, \tau)$. This is achieved by 


$$
\overline{\widehat{U}}=\left(\begin{array}{cc}
e^{i \phi_{L}(x, \tau) \hat{\tau}_{3} / 2} & 0 \\
0 & e^{i \phi_{R}(x, \tau) \hat{\tau}_{3} / 2}
\end{array}\right)
$$

Thus the $L$ diagonal element of $\overline{\widehat{U}} \overline{\widehat{G}}^{-1} \overline{\widehat{U}}^{-1}$ becomes

$$
\begin{aligned}
\hat{G}_{L}^{-1}= & -\hbar \frac{\partial}{\partial \tau} \hat{1}+i \hbar\left(\overrightarrow{\mathrm{v}}_{S L} \cdot \vec{\nabla}\right) \hat{1} \\
& +\left[\frac{\hbar^{2} \nabla^{2}}{2 m}+\mu-\frac{m}{2} \overrightarrow{\mathrm{v}}_{S L}^{2}+i\left[\frac{\hbar}{2} \frac{\partial \phi_{L}}{\partial \tau}-e V_{L}\right)\right] \widehat{\tau}_{3} \\
& \left.-\widehat{\Delta}_{L}\right\} \delta\left(x-x^{\prime}\right) \delta\left(\tau-\tau^{\prime}\right)
\end{aligned}
$$

whereas the right-left off-diagonal elements acquire a phase dependence

$$
\widehat{T}_{x x^{\prime}}=\left(\begin{array}{cc}
T_{x x^{\prime}} e^{+i \phi(\tau) / 2} & 0 \\
0 & -T_{x x^{\prime}}^{*} e^{-i \phi(\tau) / 2}
\end{array}\right) .
$$

Since $T_{x x^{\prime}}$ is finite only for $x, x^{\prime}$ near the junction (at $x=0$ ), the phase difference across the junction

$$
\phi(\tau)=\phi_{L}(x \rightarrow-0, \tau)-\phi_{R}(x \rightarrow+0, \tau)
$$

enters. Above we introduced the gauge invariant superfluid velocity

$$
\overrightarrow{\mathrm{v}}_{S L}=-\frac{\hbar}{2 m}\left[\vec{\nabla} \phi_{L}+\frac{2 e}{\hbar c} \overrightarrow{\mathrm{A}}\right] .
$$

Also the time derivative $\partial \phi_{L(R)} / \partial \tau$ appears in a gauge invariant combination in conjunction with $V_{L(R)}=(\stackrel{+}{-}) V / 2$.

What we have achieved so far is only a formal rewriting of the partition function of the two-coupled superconductor as a multiple path integral. However, we have made explicit the essential variables: the order parameters, the superfluid velocity, the phase difference, and voltage across the junction. The virtue of the path-integral representation is the systematic approximation scheme it suggests. The most probable, i.e., least action path yields classical or mean-field solutions. Beyond that, it allows us to calculate correlations of fluctuations in a straightforward way.

As a first example we consider a single homogeneous superconductor and concentrate on the magnitude of the order parameter. In this case, the action simplifies to (see Ref. 4 for more details)

$$
S_{0} / \hbar=-\sum_{k, v} e^{\omega_{v} 0^{+}} \ln \left(\omega_{v}^{2}-E_{k}^{2}\right)+\frac{\mathscr{V} \beta}{g} \Delta^{2},
$$

where $\omega_{v}$ are the complex Matsubara frequencies $\omega_{v}=i(2 v+1) \pi / \beta \hbar$ and

$$
E_{k}^{2}=\epsilon_{k}^{2}+\Delta^{2}=\left[\frac{\hbar^{2} k^{2}}{2 m}-\mu\right]+\Delta^{2}
$$

The least-action condition $\delta S_{0} / \delta \Delta=0$ reproduces the familiar BCS gap equation ${ }^{9}$

$$
1=N(0) g \int_{-\hbar \omega_{D}}^{\hbar \omega_{D}} d \epsilon_{k} \frac{1}{2 E_{k}(\bar{\Delta})} \tanh \frac{1}{2} \beta E_{k}(\bar{\Delta}),
$$

which yields the equilibrium value $\bar{\Delta}$. For an estimate of the fluctuation of $\Delta-\bar{\Delta}$ we need

$\left.\frac{\delta^{2} S_{0}}{\delta \Delta^{2}}\right|_{\Delta=\Sigma}=-\Delta \frac{\partial}{\partial \Delta}[N(0) \beta \mathscr{V}$

$$
\left.\times \int d \epsilon_{k} \frac{1}{2 E_{k}} \tanh \frac{1}{2} \beta E_{k}\right]\left.\right|_{\Delta=\Delta}
$$

We thus see that bulk energies proportional to $N(0) \mathscr{V}$ suppress the fluctuations in $\Delta$ except in a miniscule critical region $\left(T_{c}-T\right) / T_{c} \leq\left(k T_{c} / \epsilon_{F}\right)^{4}$ below the transition temperature. ${ }^{14-17}$

The effect of the superfluid velocity becomes transparent if we expand $\operatorname{Tr} \ln G^{-1}$ in $v_{S}$ to second order, using the relation

$$
\begin{aligned}
\operatorname{Tr} \ln \overline{\hat{G}}^{-1}= & \operatorname{Tr} \ln \left(\overline{\hat{G}}_{0}^{-1}+\delta \overline{\widehat{G}}^{-1}\right) \\
= & \operatorname{Tr} \ln \overline{\widehat{G}}_{0}^{-1}+\operatorname{Tr}\left(\overline{\widehat{G}}_{0} \delta \overline{\widehat{G}}^{-1}\right) \\
& -\frac{1}{2} \operatorname{Tr}\left(\overline{\widehat{G}}_{0} \delta \overline{\widehat{G}}^{-1} \overline{\widehat{G}}_{0} \delta \overline{\widehat{G}}^{-1}\right)+\cdots
\end{aligned}
$$

which is valid for general matrices. As a result we find (still considering a single superconductor) a contribution to the action

$$
\begin{aligned}
S_{1}\left(v_{s}\right) / \hbar= & \operatorname{Tr} \int \frac{d^{3} p}{(2 \pi \hbar)^{3}} \int \frac{d^{3} q}{(2 \pi \hbar)^{3}}(\hbar \beta)^{-2} \sum_{\omega_{v}, \omega_{0}}\left[\hat{G}_{0}\left[\overrightarrow{\mathrm{p}}+\frac{\overrightarrow{\mathrm{q}}}{2}, \omega_{v}+\frac{\omega_{0}}{2}\right] \hat{G}_{0}\left[\overrightarrow{\mathrm{p}}-\frac{\overrightarrow{\mathrm{q}}}{2}, \omega_{v}-\frac{\omega_{0}}{2}\right] \frac{1}{2}\left[\overrightarrow{\mathrm{p}} \cdot \overrightarrow{\mathrm{v}}_{S}\left(\overrightarrow{\mathrm{q}}, \omega_{0}\right)\right]\right. \\
& \left.\times\left[\overrightarrow{\mathrm{p}} \cdot \overrightarrow{\mathrm{v}}_{S}\left(-\overrightarrow{\mathrm{q}},-\omega_{0}\right)\right]+\frac{m}{2} \hat{\tau}_{3} \hat{G}_{0}\left(\overrightarrow{\mathrm{p}}, \omega_{v}\right) \overrightarrow{\mathrm{v}}_{S}\left(\overrightarrow{\mathrm{q}}, \omega_{0}\right) \cdot \overrightarrow{\mathrm{v}}_{S}\left(-\overrightarrow{\mathrm{q}},-\omega_{0}\right)\right] \\
= & \frac{m N}{2} \int \frac{d^{3} q}{(2 \pi \hbar)^{3}}(\hbar \beta)^{-1} \sum_{\omega_{0}} Q\left(\overrightarrow{\mathrm{q}}, \omega_{0}\right)\left|\overrightarrow{\mathrm{v}}_{S}\left(\overrightarrow{\mathrm{q}}, \omega_{0}\right)\right|^{2} .
\end{aligned}
$$

The second form defines the response kernel $Q\left(q, \omega_{0}\right)$, which relates the supercurrent to the superfluid velocity or vector potential. ( $N$ is the total density of electrons.) If $v_{S}$ varies slowly in space and time the action becomes

$$
S / \hbar=S_{0} / \hbar+\int_{0}^{\hbar \beta} \frac{d \tau}{\hbar} \int d^{3} x\left(\frac{m}{2} \rho_{S} \overrightarrow{\mathrm{v}}_{S}^{2}(x, \tau)+\frac{1}{8 \pi}\left(\overrightarrow{\mathrm{h}}-\overrightarrow{\mathrm{h}}_{\mathrm{ext}}\right)^{2}\right)
$$


with the superfluid density given by $\rho_{S}=N Q\left(q \rightarrow 0, \omega_{0}=0\right)$.

From the condition that the action be an extremum in the vector potential $\overrightarrow{\mathrm{A}}$ it follows that

$$
0=\frac{\delta S}{\delta \overrightarrow{\mathrm{A}}}=-\frac{e}{c} \rho_{S} \overrightarrow{\mathrm{v}}_{S}-\frac{1}{4 \pi} \operatorname{curl} \operatorname{curl}\left(\overrightarrow{\mathrm{A}}-\overrightarrow{\mathrm{A}}_{\mathrm{ext}}\right),
$$

which, combined with Maxwell's equation, in regions where $j_{\mathrm{ext}}=0$, yields the Meissner effect

$$
\Delta \overrightarrow{\mathrm{j}}_{S}-\lambda^{2} \overrightarrow{\mathrm{j}}_{S}=0 \text {. }
$$

From here it is a short way to flux quantization in superconducting loops of thickness much larger than the penetration depth $\lambda$. And in a SQUID geometry, where the loop contains a tunnel junction, the flux $\Phi$ through the loop and the phase difference $\phi$ across the junction are related by

$$
\Phi+\Phi_{0}(\phi / 2 \pi)=n \Phi_{0},
$$

where $\Phi_{0}$ is the flux quantum and $n$ an integer.

Expanding the action in $\left[(\hbar / 2)\left(\partial \phi_{L} / \partial \tau\right)-e V_{L}\right]$ (or $R$ ), which first contributes in second order, shows that the action is a minimum if the time derivative of the phase is pinned to the local electric potential. Thus we recover the Josephson relation

$$
\hbar \frac{\partial \phi}{\partial \tau}=2 e V .
$$

Both in the case of the Meissner effect and the Josephson relation, bulk energies suppress deviations from these meanfield results, just as they did for fluctuations in $|\Delta|$.

From the previous analysis we learn that we can restrict our attention in the following to the only remaining essential variable: the phase difference across the junction $\phi$, or equivalently by (22) if we consider a SQUID the flux through the superconducting loop. Hence the partition function of a junction, up to irrelevant factors, becomes

$$
Z_{G} \sim \int D \phi \exp \left[-\frac{S_{T}[\phi]}{\hbar}-\int_{0}^{\hbar \beta} \frac{d \tau}{\hbar} \frac{C}{2}\left[\frac{\hbar}{2 e} \frac{\partial \phi}{\partial \tau}\right]^{2}\right] .
$$

We evaluate the action explicitly by expanding in the tunnel matrix elements, which yields ${ }^{3,18}$

$$
\frac{S_{T}[\phi]}{\hbar}=-\int_{0}^{\hbar \beta} d \tau \int_{0}^{\hbar \beta} d \tau^{\prime}\left(\alpha\left(\tau-\tau^{\prime}\right) \cos \frac{\phi(\tau)-\phi\left(\tau^{\prime}\right)}{2}-\beta\left(\tau-\tau^{\prime}\right) \cos \frac{\phi(\tau)+\phi\left(\tau^{\prime}\right)}{2}\right) .
$$

The kernels $\alpha$ and $\beta$ are

$$
\begin{aligned}
& \alpha(\tau)=-\frac{2|T|^{2}}{\hbar^{2}} \int \frac{d^{3} p_{L}}{(2 \pi \hbar)^{3}} \int \frac{d^{3} p_{R}}{(2 \pi \hbar)^{3}} G_{L}\left(\tau, \overrightarrow{\mathrm{p}}_{L}\right) G_{R}\left(-\tau, \overrightarrow{\mathrm{p}}_{R}\right), \\
& \beta(\tau)=-\frac{2|T|^{2}}{\hbar^{2}} \int \frac{d^{3} p_{L}}{(2 \pi \hbar)^{3}} \int \frac{d^{3} p_{R}}{(2 \pi \hbar)^{3}} F_{L}\left(\tau, \overrightarrow{\mathrm{p}}_{L}\right) F_{R}\left(-\tau, \overrightarrow{\mathrm{p}}_{R}\right) .
\end{aligned}
$$

We made the usual assumption that the tunneling matrix element depends weakly on the momenta near $p_{F}$. The Green's functions $G$ and $F$ are the diagonal $(1,1)$ and off-diagonal $(1,2)$ components of the Nambu-Green's function, whose inverse is given by Eq. (13). To the extent that the tunneling of the electrons distorts the electrodes only weakly, we can take for $G$ and $F$ the equilibrium functions

$$
G\left(\omega_{v}, \overrightarrow{\mathrm{p}}\right)=-\hbar\left(i \hbar \omega_{v}+\epsilon_{p}\right) /\left[\left(\hbar \omega_{v}\right)^{2}+E_{p}^{2}\right]
$$

and

$$
F\left(\omega_{v}, \overrightarrow{\mathrm{p}}\right)=\hbar \Delta /\left[\left(\hbar \omega_{v}\right)^{2}+E_{p}^{2}\right] .
$$

The momentum integrations in (26) are now easily performed, which yields in the range $-\hbar \beta \leq \tau \leq \hbar \beta$

$$
\int \frac{d^{3} p}{(2 \pi \hbar)^{3}}\left[\begin{array}{l}
\boldsymbol{G} \\
\boldsymbol{F}
\end{array}\right](\tau, p)=-N(0) \int_{-\infty}^{\infty} \mathscr{N}(E)\left[\begin{array}{c}
1 \\
\Delta / E
\end{array}\right] e^{-E \tau / \hbar}\left[\left(1-f_{E}\right) \Theta(\tau)-f_{E} \Theta(-\tau)\right] .
$$

Above $f_{E}$ denotes the Fermi function, and $\mathscr{N}(E)=|E| \Theta(|E|-\Delta) /\left(E^{2}-\Delta^{2}\right)^{1 / 2}$ is the BCS density of states.

The action (25) with $\alpha$ and $\beta$ given by (26) provides a general description of the quantum-statistical properties of a Josephson tunnel junction. We will now proceed and in the remainder of this section discuss various limits or approximations. 
At zero temperature the integration in (27) is readily performed. We find

$$
\begin{aligned}
& \alpha(\tau)=\frac{\hbar}{2 \pi e^{2} R_{N}} \frac{\Delta_{L} \Delta_{R}}{\hbar^{2}} K_{1}\left(\frac{\Delta_{L}|\tau|}{\hbar}\right) K_{1}\left(\frac{\Delta_{R}|\tau|}{\hbar}\right), \\
& \beta(\tau)=\frac{-\hbar}{2 \pi e^{2} R_{N}} \frac{\Delta_{L} \Delta_{R}}{\hbar^{2}} K_{0}\left(\frac{\Delta_{L}|\tau|}{\hbar}\right) K_{0}\left(\frac{\Delta_{R}|\tau|}{\hbar}\right),
\end{aligned}
$$

where $K_{0}$ and $K_{1}$ are modified Bessel functions. The prefactor involves the normal state (or high voltage) conductance of the junction $1 / R_{N}=4 \pi e^{2}|T|^{2} N_{L}(0) N_{R}(0) / \hbar$. The limiting behavior of $\alpha$ for equal gaps $\Delta_{L}=\Delta_{R}=\Delta$ is

$$
\alpha(\tau) \approx \frac{\hbar}{2 \pi e^{2} R_{N}}\left\{\begin{array}{l}
1 / \tau^{2} \text { for }|\tau| \ll \hbar / \Delta \\
\left.\frac{\pi}{2}\left[\frac{\Delta}{\hbar}\right]^{2} \frac{\hbar}{\Delta|\tau|} e^{-(2 \Delta|\tau| / \hbar)} \text { for }|\tau| \gg\right\rangle / \Delta
\end{array}\right.
$$

and $\beta(\tau)$ has the same long-time expansion, but is proportional to $\ln ^{2} \tau$ for small $\Delta|\tau| / \hbar$. Another interesting case is the limit where $\Delta$ vanishes. Then for general temperatures

$$
\alpha(\tau)=\frac{\hbar}{2 \pi e^{2} R_{N}} \frac{(\pi k T / \hbar)^{2}}{\sin ^{2}(\pi k T \tau / \hbar)} \quad(\Delta=0) .
$$

The physics of the terms involving $\alpha$ and $\beta$ is quite different. Suppose $\phi(\tau)$ varies slowly on the time scale determined by the extent of $\alpha$ and $\beta$, namely $\hbar / \Delta$. This can be arranged by appropriate choice of junction parameters. In this limit the $\beta$ term becomes local in time. We observe, furthermore, that $\beta$ is related to the critical current of the junction $\int_{-\hbar \beta / 2}^{\pi \beta / 2} d \tau \beta(\tau)=-I_{c} / 2 e$. Hence the $\beta$ term (in lowest order) gives rise to the "washboard potential"

$$
S_{\beta} / \hbar=-\int_{0}^{\hbar \beta} d \tau\left(I_{c} / 2 e\right) \cos \phi(\tau)
$$

In contrast the $\alpha$ term has a leading $\phi$ dependence involving $(\partial \phi / \partial \tau)^{2}$. We will exploit this property further below.

If the superconducting tunnel junction is biased by an external current $I$, we have to add an additional potential contribution

$$
S_{I} / \hbar=-\int_{0}^{\hbar \beta} d \tau(I / 2 e) \phi,
$$

which tilts the washboard. This term can be justified from the kinetic energy terms $(m / 2) \rho_{S L(R)} \overrightarrow{\mathbf{v}}_{S L(R)}^{2}$ [see Eq. (19)] integrated over the $L$ (or $R$ ) electrode with suitable boundary conditions "at the battery." A more precisely defined model of the current source, taking care of the boundary condition, is achieved by closing the electrodes in a large loop, with a large number of windings of the phase around the loop. This, of course, represents a SQUID with a large trapped flux. In this case the kinetic energy, integrated around the loop gives rise to the extra potential contribution $S_{I}$. Since $I$ is the current in the bulk, it can be considered constant even if $\phi$ changes by amounts of order $2 \pi$.

At this level of approximation, ignoring a constant term, we can write the effective action of a current biased tunnel junction as $^{3}$

$$
\begin{array}{r}
S[\phi] / \hbar=\int_{0}^{\hbar \beta} \frac{d \tau}{\hbar}\left[\frac{C}{2}\left[\frac{\hbar}{2 e} \frac{\partial \phi}{\partial \tau}\right]^{2}+U(\phi)\right] \\
+2 \int_{0}^{\hbar \beta} d \tau \int_{0}^{\hbar \beta} d \tau^{\prime} \alpha\left(\tau-\tau^{\prime}\right) \\
\times \sin ^{2}\left\{\frac{1}{4}\left[\phi(\tau)-\phi\left(\tau^{\prime}\right)\right]\right\}
\end{array}
$$

with a tilted washboard potential

$$
U(\phi)=-\frac{I_{c} \hbar}{2 e} \cos \phi-\frac{I \hbar}{2 e} \phi
$$

The action of a SQUID differs in the potential term by the magnetic field energy. Hence

$$
U(\Phi)=-\frac{I_{c} \hbar}{2 e} \cos \left[2 \pi \frac{\Phi}{\Phi_{0}}\right]+\frac{1}{2 L}\left(\Phi-\Phi_{\mathrm{ext}}\right)^{2}
$$

where $L$ is the geometric inductance. Also in the kinetic (capacitive) energy and the $\alpha$ term we should express the phase $\phi$ by the flux $\Phi$ using relation (22).

The action in the form (32) bears close similarity to that of Caldeira and Leggett., ${ }^{5,6}$ In particular, the $\alpha$ term represents the effect of damping. In our model it is due to quasiparticle tunneling across the barrier. We will draw further comparisons and comment on the differences in Sec. IV. In the case where the damping is due to a normal shunt tunnel junction, which we may model by setting $\Delta=0$ in the $\alpha$ term, or at finite temperatures, the form (32) is about as far as we can simplify the action. In the case of an ideal superconducting tunnel junction at zero temperature, provided that the characteristic time scale of the motion of $\phi$ is slow compared to $\hbar / \Delta$, the short range of $\alpha$ as well as of $\beta$ allows us further approximation. Expanding $\sin ^{2}\left(\phi-\phi^{\prime}\right) / 4$ leads to

$$
S_{\alpha} / \hbar \approx \frac{1}{4} \int_{0}^{\hbar \beta} d \tau(\partial \phi / \partial \tau)^{2} \int_{0}^{\hbar \beta / 2} d \tau^{\prime} \alpha\left(\tau^{\prime}\right) \tau^{\prime 2} .
$$

In this form we see that the effects of the quasiparticles under the given conditions are to increase the effective capacitance. In a mechanical analog the particle becomes heavier. For $\Delta_{L}=\Delta_{R}$ this change is ${ }^{4,18}$

$$
\delta C / C=3 \pi \hbar /\left(32 \Delta R_{N} C\right) .
$$


A similar correction results from the $\beta$ term, if we include higher terms of the expansion in $\left(\tau-\tau^{\prime}\right)$. Combining both we find the action contains an enhanced, phase- (flux) dependent capacitance (ren is renormalized),

$$
C_{\text {ren }}=C+\delta C[1-(1 / 3) \cos \phi] \text {. }
$$

Above, we expressed the partition function in terms of a path integral, which is of interest if we want to calculate thermodynamic properties, including (in the limit $\beta=1 / k T \rightarrow \infty)$ ground-state properties. We can easily generalize our approach to include external sources. For example, the generating functional

$$
Z[\xi]=\operatorname{Tr}\left[T \exp \left[-\frac{1}{\hbar} \int_{0}^{\hbar \beta} d \tau\left[H-I_{t}(\tau) \xi(\tau)\right]\right)\right]
$$

provides a convenient, systematic formulation to express expectation values and correlation functions of the tunneling current $I_{t}$ in various situations (see, e.g., Ref. 19). We will not comment on this point further since some of the results will emerge from the real-time analysis to be presented in the next section.

\section{REAL-TIME DESCRIPTION}

In the last section we developed a quantum-statistical description of our system of interest. The statistical Boltzmann factor $e^{-\beta H}$ leads us to consider formally path integrals in imaginary times $t=-i \tau$ where $t$ varies between $-i \hbar \beta$ and 0 . In this section we will present a path-integral description in real times. The averaging over the internal degrees of freedom (quasiparticles in the case of the tunnel junction) is done on the level of probabilities and not amplitudes. This leads us to consider double pair integrals (a forward and backward propagator), which are coupled after averaging. ${ }^{20}$

As an example, we consider the time development of the density matrix $\rho(t)$. It is governed by the equation

$$
\rho\left(t_{f}\right)=U\left(t_{f}, t_{i}\right) \rho\left(t_{i}\right) U\left(t_{i}, t_{f}\right),
$$

where $U\left(t_{f}, t_{i}\right)$-the time evolution operator-is the time-ordered exponential

$$
U\left(t_{f}, t_{i}\right)=T \exp \left[-i \int_{t_{i}}^{t_{f}} d t^{\prime} H\left(t^{\prime}\right) / \hbar\right) .
$$

Again our system of interest has several degrees of freedom. We want to keep track of one of them; the rest of the degrees of the reservoir remain unobserved and should be eliminated from the description. For simplicity, let us assume first that these degrees of freedom are coordinates, denoted by $x$ and $\overrightarrow{\mathrm{R}}=R_{1}, \ldots, R_{N}$, respectively. We assume that the system is initially in "thermal equilibrium" (to be specified further in a moment) and we trace over all the final states of $\vec{R}$. We thus are lead to consider a reduced density matrix

$$
\begin{aligned}
\int d^{N} R\left\langle x_{1} \overrightarrow{\mathrm{R}}\left|\rho\left(t_{f}\right)\right| x_{2} \overrightarrow{\mathrm{R}}\right\rangle= & \int d x_{1}^{\prime} d x_{2}^{\prime} d^{N} R_{1}^{\prime} d^{N} R_{2}^{\prime} d^{N} R\left\langle x_{1} \overrightarrow{\mathrm{R}}\left|U\left(t_{f}, t_{i}\right)\right| x_{1}^{\prime} \overrightarrow{\mathrm{R}}_{1}^{\prime}\right\rangle \\
& \times\left\langle x_{1}^{\prime} \overrightarrow{\mathrm{R}}_{1}^{\prime}\left|\rho\left(t_{i}\right)\right| x_{2}^{\prime} \overrightarrow{\mathrm{R}}_{2}^{\prime}\right\rangle\left\langle x_{2}^{\prime} \overrightarrow{\mathrm{R}}_{2}^{\prime}\left|U\left(t_{i}, t_{f}\right)\right| x_{2} \overrightarrow{\mathrm{R}}\right\rangle .
\end{aligned}
$$

There remains some ambiguity in the way the initial state is prepared, differing (at least) in the resulting transient, short-time behavior. Feynman and Vernon, ${ }^{7}$ and others thereafter, $8,21,22$ assumed that at $t_{i}$ the system factorizes as $\rho\left(t_{i}\right)=\rho_{x}\left(t_{i}\right) \cdot \rho_{\overrightarrow{\mathrm{R}}}\left(t_{i}\right)$ where $\rho_{\overrightarrow{\mathrm{R}}}$ is the thermal density matrix of the reservoir by itself. This is equivalent to assuming that there was no interaction between the reservoir and the coordinate of interest $x$ before $t_{i}$. Alternatively, we may assume that the total system was thermalized, before, at $t_{i}$, we project out a certain state of $x$. Formally this means we fix the initial values of $x_{1}^{\prime}$ and $x_{2}^{\prime}$ by $\delta\left(x_{1}^{\prime}-x_{1 i}\right) \delta\left(x_{2}^{\prime}-x_{2 i}\right)$ in (37). We achieve this by a measurement of $x$ at $t_{i}$, rejecting all events which do not satisfy the initial conditions. Finally, we can imagine keeping $x$ fixed at a value $x=x_{i}$ before $t_{i}$ and having the reservoir thermalized in interaction with $x$ according to this fixed value. This possibility represents those physical situations where we prepared the system in the initial state by applying a suitable field (coupling to $x$ only) which is switched off at $t_{i}$.

We exploit the formal similarity between the statistical Boltzmann factor $\rho\left(t_{i}\right)=U\left(t_{i}-i \hbar \beta, t_{i}\right) / Z_{G}$ and the timeevaluation operator to write the reduced density matrix as

$$
\begin{aligned}
\int d^{N} R\left\langle x_{1} \overrightarrow{\mathrm{R}}\right. & \rho\left(t_{f}\right)\left|x_{2} \overrightarrow{\mathrm{R}}\right\rangle \\
& =\int d^{N} R \frac{1}{Z_{G}} \int_{C} D x D^{N} R e^{i S[x, R] / \hbar},
\end{aligned}
$$

where the path is along the Kadanoff-Baym ${ }^{23}$ contour $C$ in the complex $t$ plane connecting the points $t_{i}, t_{f}, t_{i}, t_{i}-i \hbar \beta$. The path integral in (38) is performed subject to the constraints $x=x_{1}$ at the end of the path from $t_{i}$ to $t_{f}$ and $x=x_{2}$ at the beginning of the path from $t_{f}$ to $t_{i}-i \hbar \beta$. Also, the specification of the initial "thermal state" may be expressed by further constraints on $x$ along the vertical part of the path.

To proceed we want to eliminate the reservoir degrees of freedom and obtain an effective description expressed in terms of the interesting variable $x$ only. The procedure is similar in principle to that of Sec. II. But in contrast to that problem where we calculated complete traces only, we now have to specify $x$ at various times. This is a straightforward extension, if $x$ is a particle coordinate. It also is straightforward, in the case of a SQUID, where the interesting variable is the vector potential $\vec{A}$ (or the flux, which is a linear function of $\vec{A}$ ), and the reservoir variables to be integrated out are the electron degrees of freedom, i.e., $\psi_{\sigma}(x, t)$. However, we encounter a conceptual problem in the case of a Josephson tunnel junction, where the interesting variable is the phase difference. The problem arises from the fact that we have not identified a set of simultaneously measurable variables which together with the phase completely specify the state of the system. This prevents us from projecting out states with definite phase. We circumvent this complication by considering 
the tunnel junction (again) as part of a SQUID. The Meissner effect and the flux quantization establish (on the mean-field level) a linear relation between the phase difference across the junction and the trapped flux, i.e., the vector potential $\overrightarrow{\mathrm{A}}$. In this sense we can indeed treat the phase as an ordinary variable.

We now repeat all the steps of the last section, i.e., introduce order parameters and voltage fields; however, all the path integrals are taken along the Kadanoff-Baym contour in the complex-time plane, and we restrict ourselves again to the only strongly fluctuating variable: the phase difference in the case of the tunnel junction or the flux in the SQUID. For definiteness we concentrate on the case of the tunnel junction, where

$$
\left\langle\phi_{1}\left|\rho\left(t_{f}\right)\right| \phi_{2}\right\rangle=\frac{1}{Z_{G}} \int_{C} D \phi e^{i S[\phi] / \hbar} .
$$

The resulting effective action $S[\phi]$ for real times is obtained from the corresponding imaginary-time action by an analytic continuation $\tau \rightarrow$ it. However, care has to be exercised regarding the nonlocal terms in $S$, e.g., those double-time integrals representing the dissipation. The kernels $\alpha\left(t, t^{\prime}\right)$ and $\beta\left(t, t^{\prime}\right)$ are discontinuous functions of their time arguments at $t=t^{\prime}$ if both $t$ and $t^{\prime}$ are on the same (either forward or backward) path. More precisely, if $t$ and $t^{\prime}$ are on the forward (backward) path the timeordered $\alpha^{c}\left(t, t^{\prime}\right)$ [antitime ordered $\alpha^{\bar{c}}\left(t, t^{\prime}\right)$ ] enters (similarly for $\beta)$, whereas for $t$ and $t^{\prime}$ on different paths $\alpha^{>}\left(t, t^{\prime}\right)$ or $\alpha^{<}\left(t, t^{\prime}\right)$ appear, reflecting that times on the backward path are always "later" than those on the forward path.

If we express, in the usual way, the diagonal and offdiagonal Green's function $G$ and $F$ by the spectral densities and equilibrium distribution functions, we find

$$
\begin{aligned}
& \alpha^{(<)}(t)=( \pm) \frac{1}{e} \int_{-\infty}^{\infty} \frac{d \omega}{2 \pi} e^{-i \omega t} \frac{1}{e^{(\overline{+}) \beta \hbar \omega}-1} I_{n}(\omega), \\
& \beta^{\left(<^{<}\right)}(t)=\left( \pm \frac{+}{2} \frac{1}{e} \int_{-\infty}^{\infty} \frac{d \omega}{2 \pi} e^{-i \omega t} \frac{1}{e^{(\mp) \beta \hbar \omega}-1} I_{c}(\omega) .\right.
\end{aligned}
$$

Again we assume that the distribution of the electrons, except for the fact that they instantaneously follow the shift of the electric potential, is little distorted by the tunneling of electrons. The quantity $I_{n}(\omega)$ is the quasiparticle current at voltage $\hbar \omega / e$, whereas the quantity $I_{c}$ is related to the supercurrent. They are

$$
\begin{aligned}
& \left.\begin{array}{c}
I_{n}(\omega) \\
I_{c}(\omega)
\end{array}\right\}=\frac{1}{2 e R_{N}} \int_{-\infty}^{\infty} d E \mathscr{N}\left(E+\frac{1}{2} \hbar \omega\right) \mathscr{N}\left(E-\frac{1}{2} \hbar \omega\right) \times\left\{\begin{array}{c}
1 \\
\frac{\Delta^{2}}{\left(E+\frac{1}{2} \hbar \omega\right)\left(E-\frac{1}{2} \hbar \omega\right)}
\end{array}\right\} \\
& \times\left\{\tanh \left[\left(E+\frac{1}{2} \hbar \omega\right) / 2 k T\right]-\tanh \left[\left(E-\frac{1}{2} \hbar \omega\right) / 2 k T\right]\right\},
\end{aligned}
$$

where $\mathscr{N}(E)=\Theta(|E|-\Delta)|E| /\left(E^{2}-\Delta^{2}\right)^{1 / 2}$ is the BCS density of states. The zero voltage critical supercurrent is given as

$$
I_{c}=\mathrm{P} \int_{-\infty}^{\infty} \frac{d \omega}{\pi} \frac{I_{c}(\omega)}{\omega}
$$

For real times $t, \alpha$ has real and imaginary parts $\alpha^{\left(\gtrless^{\prime}\right)}(t)=\alpha_{R}(t)(\stackrel{+}{-}) i \alpha_{I}(t)$ and

$$
\alpha^{c(\bar{c})}(t)=\alpha_{R}(t)(\stackrel{+}{-}) i \operatorname{sgn}(t) \alpha_{I}(t) .
$$

Corresponding relations hold for $\beta$. We recall the fact that $\alpha$ and $\beta$ are products of two Green's functions. From this it is apparent that $\alpha_{I}\left(\beta_{I}\right)$ has the meaning of a junction response function, whereas $\alpha_{R}\left(\beta_{R}\right)$ has the meaning of a correlation function. Indeed, they are related by the fluctuation-dissipation theorem

$$
\alpha_{R}(\omega)=i \alpha_{I}(\omega) \operatorname{coth} \frac{\hbar \omega}{2 k T}
$$

with a similar relation for $\beta$.

To put (39) into a more transparent form it is convenient to distinguish between the forward and backward paths connecting $t_{i}$ and $t_{f}$. Calling the path function $\phi_{1}(t)$ on the forward segment from $t_{i}$ to $t_{f}$ and $\phi_{2}(t)$ on the backward segment we can write the real-time segments of (39) as a double path integral

$$
\int D \phi_{1} D \phi_{2} e^{i S\left[\phi_{1}, \phi_{2}\right] / \hbar}
$$

where both $\phi_{1}(t)$ and $\phi_{2}(t)$ are functions over the domain $t_{i} \leq t \leq t_{f}$. Equation (44) is of the Feynman-Vernon form. ${ }^{7}$ Following Schmid, ${ }^{8}$ who discussed a similar problem of a particle coupled to harmonic oscillators, we introduce a "center-of-mass" and "relative" coordinate $\phi=\frac{1}{2}\left(\phi_{1}+\phi_{2}\right)$ and $\chi=\phi_{1}-\phi_{2}$. In terms of these variables the effective action, which is complex $S=S_{1}+i S_{2}$, becomes ${ }^{4,18}$ 


$$
\begin{aligned}
S_{1}[\phi, \chi]= & \int_{t_{i}}^{t_{f}} d t\left[C\left[\frac{\hbar \dot{\phi}}{2 e}\right]\left[\frac{\hbar \chi}{2 e}\right]+I\left[\frac{\hbar \chi}{2 e}\right]\right] \\
& +8 \hbar \int_{t_{i}}^{t_{f}} d t \int_{t_{i}}^{t_{f}} d t^{\prime}\left[\alpha_{I}\left(t-t^{\prime}\right) \sin \frac{\phi-\phi^{\prime}}{2}-\beta_{I}\left(t-t^{\prime}\right) \sin \frac{\phi+\phi^{\prime}}{2}\right] \Theta\left(t-t^{\prime}\right) \sin \frac{\chi}{4} \cos \frac{\chi^{\prime}}{4}, \\
S_{2}[\phi, x]= & 4 \hbar \int_{t_{i}}^{t_{f}} d t \int_{t_{i}}^{t_{f}} d t^{\prime}\left[\alpha_{R}\left(t-t^{\prime}\right) \cos \frac{\phi-\phi^{\prime}}{2}+\beta_{R}\left(t-t^{\prime}\right) \cos \frac{\phi+\phi^{\prime}}{2}\right] \sin \frac{\chi}{4} \sin \frac{\chi^{\prime}}{4} .
\end{aligned}
$$

It is possible to interpret $S_{2}$, the imaginary part of the action, as the result of Gaussian stochastic force. In the present problem some care has to be exercised because of the factor $\cos \left(\phi \mp \phi^{\prime}\right) / 2$ multiplying $\alpha_{R}\left(\beta_{R}\right)$. The inverse of $\alpha_{R}\left(t-t^{\prime}\right) \cos \left(\phi-\phi^{\prime}\right) / 2$ is not a positive definite operator. We overcome this complication by splitting $S_{2}$ into two parts and introducing two independent stochastic forces $\xi_{1}$ and $\xi_{2}$, such that

$$
\begin{aligned}
& e^{-S_{2} / \hbar}=\left\langle e^{i S_{\xi} / \hbar}\right\rangle_{\xi_{1}, \xi_{2}}, \\
& S_{\xi} / \hbar=\frac{2}{e} \int_{t_{i}}^{t_{f}} d t\left[\xi_{1}(t) \cos \frac{\phi}{2}+\xi_{2}(t) \sin \frac{\phi}{2}\right] \sin \frac{\chi}{4} .
\end{aligned}
$$

The average is taken over the two independent Gaussian random variables, $\xi_{1}$ and $\xi_{2}$, with autocorrelation functions

$$
\begin{aligned}
& \left\langle\xi_{1(2)}(t) \xi_{1(2)}\left(t^{\prime}\right)\right\rangle=2 e^{2}\left[\alpha_{R}\left(t-t^{\prime}\right)(\stackrel{+}{-}) \beta_{R}\left(t-t^{\prime}\right)\right], \\
& \left\langle\xi_{1}(t) \xi_{2}\left(t^{\prime}\right)\right\rangle=0,
\end{aligned}
$$

where $\alpha_{R}$ and $\beta_{R}$ follow from Eq. (40).

The action (45), with the possible interpretation of $S_{2}$ as given in (46), represents a general description of the dynamics of a Josephson tunnel junction (or with suitable extension of a SQUID). Frequently it is sufficient to consider simpler approximate versions. For example, in many cases the variations of the phase are slow on a time scale given by $\hbar / \Delta$. Then $\beta_{I}$ dominates over $\beta_{R}$ and to lowest order can be approximated by $\beta_{I}=\left(I_{c} / 2 e\right) \delta\left(t-t^{\prime}\right)$. Substituting this into (45) yields the contribution

$$
\int d t\left(I_{c} \hbar / 2 e\right)[\cos (\phi+\chi / 2)-\cos (\phi-\chi / 2)],
$$

i.e., exactly the effect of a washboard potential $U(\phi)=-\left(I_{c} \hbar / 2 e\right) \cos \phi$. Consistently, the correlation functions of $\xi_{1}$ and of $\xi_{2}$ are identical, given by $\alpha_{R}$ only.

At $T=0$, in the superconducting tunnel junction model, the effect of the environment for slow phase motions in real time is, as in the description of Sec. II, to modify the capacitance. Keeping the lowest nonvanishing time moments of the nonlocal terms in (45)-the zeroth and second moments of $\beta_{I}$ and the second moment of $\alpha_{I}$-which can be calculated exactly in this model, one finds the correction equivalent to using Eqs. (33) and (34) in the forward and backward time-evolution operators. Thus all the slow physics of the superconducting tunnel junction at zero temperature can be obtained from a Schrödinger equation.

The response function $\alpha_{I}$ and the correlation function $\alpha_{R}$ in general depend on $\Delta$ reflecting the gap dependence of the quasiparticle current. Nevertheless, it is instructive to consider them in the limit $\Delta=0$, while retaining the washboard potential (i.e., retaining the supercurrent $I_{c} \neq 0$ ). We can think about this limit as describing a Josephson junction shunted by a normal tunnel junction with conductance much larger than that of the Josephson junction. In this limit the normal current is purely Ohmic $I_{N}(\omega)=\hbar \omega /\left(e R_{N}\right)$. Hence

$$
\begin{aligned}
& \alpha_{I}^{N}(t)=\hbar /\left(2 e^{2} R_{N}\right) \frac{\partial}{\partial t} \delta(t), \\
& \alpha_{R}^{N}(\omega)=\hbar \omega /\left(2 e^{2} R_{N}\right) \operatorname{coth}(\beta \hbar \omega / 2) .
\end{aligned}
$$

Within this approximation the total action becomes

$$
\begin{gathered}
S_{1}=\int_{t_{i}}^{t_{f}} d t\left[C \frac{\hbar \dot{\phi}}{2 e} \frac{\hbar \dot{\chi}}{2 e}-\frac{1}{R_{N}} \frac{\hbar \dot{\phi}}{2 e} \frac{\hbar}{e} \sin \frac{\chi}{2}\right. \\
-U(\phi+\chi / 2)+U(\phi-\chi / 2)], \\
S_{2}=4 \hbar \int_{t_{i}}^{t_{f}} \int_{t_{i}}^{t_{f}} d t d t^{\prime} \sin \frac{\chi}{4} \alpha_{R}^{N}\left(t-t^{\prime}\right) \cos \frac{\phi-\phi^{\prime}}{2} \sin \frac{\chi^{\prime}}{4} .
\end{gathered}
$$

Finally, we mention that if we replace the trigonometric functions in (49) by their small argument expansions we reproduce the effective action found in Refs. 8 and 21, which describes a particle coupled linearly to a suitable set of harmonic oscillators.

\section{RESULTS, COMPARISON, AND DISCUSSION}

In the previous two sections we developed the formal theory describing a superconducting tunnel junction. In the following we will first summarize what we have done so far and then draw conclusions and discuss results. We will also compare with related work on simpler systems.

\section{A. Summary}

We showed, starting from the microscopic theory, i.e., the BCS theory of superconductivity and a tunnel Hamiltonian for the tunnel junction, how the relevant degrees of freedom, i.e., the complex order parameter of the superconductors and the voltage difference across the junction arise. By introducing them as variables in a functional or path integral we do not limit ourselves to a self-consistent field theory. All the other electronic degrees of freedom are assumed to adjust to these fields. We eliminate them by taking the trace over the fermion field operators. We found that fluctuations in the magnitude of the order pa- 
rameter, as well as deviations from the Josephson relation $\hbar \dot{\phi}=2 e V$ and the Meissner effect are suppressed by bulk energies. In contrast the phase difference $\phi$ feels only a shallow potential proportional to the critical current $I_{c}$ of the junction, which is small since it itself depends on the microscopic tunnel matrix element.

We obtain explicit results if we treat the tunneling in the usual approximation scheme, i.e., assume that the matrix element $T_{x x^{\prime}}$ is small and weakly dependent on the momentum transfer. Our main results are the effective (imaginary time) action (25) from which the quantumstatistical properties can be derived, and the double field, real-time action (45) which governs the time evolution of the system. Our description contains the standard theory of electron tunneling in Josephson junctions, i.e., both the supercurrent, the quasiparticle tunneling current, and quasiparticle-supercurrent interference terms. We will demonstrate this below.

At this stage a comment on the physical consequence of our small $T$ expansion is in order. We explicitly keep track of the total charge of the electrons in each electrode. However, we assume (when evaluating $\alpha$ and $\beta$ ) that the energy distribution of the electrons in the electrodes is thermal-even though the tunnel process at finite voltages may lead to a population change far away from the Fermi level. Clearly this assumption is justified if there exists a fast relaxation process. The distortion of the distribution function created by the tunneling in principle modifies the tunnel current. But we estimate this effect to be very small as long as the tunnel current is weak and the electrodes are not of microscopic dimensions. The fact that we are interested in variations on time scales short compared to inelastic lifetimes demonstrates the relevance of the second argument. On the other hand, it is the eventual thermalization of the electrons (by emission of phonons which may escape into the heat bath) that removes the energy dissipated in the charge-transfer processes from the electronic system. As is typical for transport phenomena, we can proceed, calculating response functions in low order whereas the dissipated energy becomes relevant only in higher order.

In those circumstances, where the phase varies slowly on a time scale given by $\hbar / \Delta$, the short range of $\beta(\tau)$ [or of $\beta_{I}(t)$ ] allows us to replace the $\beta$ terms by a potential $-\left(I_{c} \hbar / 2 e\right) \cos \phi$. This approximation corresponds to replacing the supercurrent by $I_{S}=I_{c} \sin \phi(t)$. (The general expression will be recovered below.) Although this approximation is strictly true only for constant $\phi$, experience tells us that this is a good qualitative approximation for most problems. The resulting imaginary-time action is now of a rather similar structure as that of Caldeira and Leggett. ${ }^{5,6}$ However, there are still-even qualitativedifferences in the dissipation term. The model of Caldeira and Leggett-a particle interacting linearly with a suitably chosen reservoir of harmonic oscillator [linear both in the particle $(x)$ and reservoir $\left(R_{i}\right)$ coordinates, i.e., $\left.H_{\mathrm{int}}=x \sum_{i} C_{i} R_{i}\right]$-was constructed to produce in the classical limit a velocity proportional, i.e., Ohmic damping. In our system the dissipation results from the tunneling of quasiparticles. The gap dependence of $\alpha$ and the sinusoidal phase dependence reflect the peculiarities of the quasiparticle current in a Josephson junction. Taking the $\Delta=0$ limit of $\alpha$ [i.e., using (29) or (49)] models a normal tunnel junction, which has an Ohmic dissipation. In this case, at zero temperature $\alpha(\tau) \sim 1 / \tau^{2}$. This result agrees with the kernel of Caldeira and Leggett, who obtained it by choosing the spectral density of the harmonic oscillators and coupling strength $C_{i}$ suitably. Clearly, for a different choice of these parameters, within the linearcoupling model, any functional dependence of $\alpha(\tau)$ can be simulated. ${ }^{24}$ It is interesting to note that the dissipation of a particle due to interaction with particle-hole pairs of a Fermi fluid also results in a kernel $\alpha(\tau) \sim 1 / \tau^{2}$ (see Refs. 25).

We furthermore draw attention to the fact that our variable is a phase which enters into our action only as derivative or in trigonometric functions (with the exception of the approximated term representing the current source). The resulting cyclicity of the action reflects the discreteness of the underlying charge-transfer process. As a result (which will be demonstrated below) the noise of the tunnel junction, normal or superconducting, is shot noise. In this respect our result differs from that of Caldeira and Leggett even in the $\Delta=0$ limit. Their variable is a coordinate and the noise obtained from their analysis is ordinary Gaussian, i.e., Johnson-Nyquist noise. 8,21 Their model for dissipation describes an ordinary resistor. Formally our description reduces to theirs if, in addition to setting $\Delta=0$, we replace in the $\alpha$ terms the trigonometric functions by their small argument expansions. Conversely, the phase-related features of our model can be obtained by a simple generalization of Caldeira and Leggett's model: We have to couple to two independent ensembles of harmonic oscillators $\left\{R_{i}^{(1)}\right\}$ and $\left\{R_{i}^{(2)}\right\}$ by a coupling of the form

$$
H_{\mathrm{int}}=\cos (\phi / 2) \sum_{i} C_{i}^{(1)} R_{i}^{(1)}+\sin (\phi / 2) \sum_{i} C_{i}^{(2)} R_{i}^{(2)} .
$$

The coupling strengths $C_{i}^{(1)}$ and $C_{i}^{(2)}$ and the spectral densities of the oscillators are related to $\alpha(\tau) \pm \beta(\tau)$.

Finally, we note that in our description the tunneling Hamiltonian yields both the dissipation related to the quasiparticle current and the potential corresponding to the supercurrent. Therefore, questions of potential renormalization never arise. Such questions arose in connection with the model of Caldeira and Leggett and caused some misunderstanding in the literature. ${ }^{26}$ Obviously, also in their model a careful physical interpretation settles this question in an unambiguous way. ${ }^{6}$

\section{B. Quantum Langevin equations}

The time evolution of the density matrix discussed in Sec. III contains all the quantum-mechanical information on our system. It may serve as a starting point to analyze the effect of dissipation on quantum coherence (compare Chakravarty and Leggett ${ }^{22}$ ) or quantum interference (compare Caldeira and Leggett ${ }^{27}$ ). So far, this general theory has been applied only in very simple systems as, for example, a two-level system, or for a harmonic oscillator. Two approximation schemes appear useful to proceed: In certain cases, if the nonlocality of the correla- 
tion functions $\alpha_{R}, \beta_{R}$ is irrelevant, we can derive a Fokker-Planck description (see, e.g., Ref. 21). Alternatively, we may treat the variable of interest, i.e., in our case the phase $\phi$, as a classical variable. The coupling to the reservoir, i.e., the quasiparticle degrees of freedom, leads to dissipation and noise. Thus in the classical limit the equation of motion is a Langevin equation. In this limit $(\hbar \rightarrow 0)$ the equation of motion can be derived from the least action principle

$$
\left.\frac{\delta S\left[\phi, \chi, \xi_{i}\right]}{\delta \chi}\right|_{\chi=0}=0 .
$$

Here $S\left[\phi, \chi, \xi_{i}\right]=S_{1}\left[\phi_{1} \chi\right]+S_{\xi}$ where $S_{1}$ is the real part of the action (45) (in the two fields) and $S_{\xi}$ was introduced in (46) replacing the imaginary part $S_{2}$. The fields $\xi_{1}$ and $\xi_{2}$ are Gaussian stochastic variables with correlation functions given by the $\hbar \rightarrow 0$ limit of (47). (Notice that $\chi=0$ is a solution of $d S / d \phi=0$.) We do not write the Langevin equation at this stage (it will be written below). Here we merely mention that the noise term is $\delta$ correlated with amplitude proportional to $2 k T / R$.

There have been attempts in the past to extend phenomenologically the Langevin equation beyond the strictly classical regime. ${ }^{28} \mathrm{~A}$ partial formal justification was given by Schmid. ${ }^{8}$ The form of this "quantum Langevin equation" is the same as of the classical counterpart; however, the noise is "quantum noise, reflecting the quantum nature of the reservoir. In simple cases the noise correlation function is $(\hbar \omega / R) \operatorname{coth}(\hbar \omega / 2 k T)$, which in the classical limit $\hbar \omega<<k T$ reduces to the classical white-noise spectrum. Technically, this quantum Langevin equation follows from the condition (50) generalized in the respect that the correlation functions are retained in their general $\hbar$-dependent form. Although this extension appears suggestive, we stress that the question of its validity is not settled, unless the system is purely harmonic $^{29}$ (see also Refs. 30 and 31 for further comments). We will follow this procedure anyhow and present here the quantum Langevin equation of an ideal tunnel junction. We do so in order to point out the interesting differences to the ordinary quantum Langevin equation, which was applied to describe resistively shunted junctions. We will also demonstrate that in certain limits-other than $\hbar \rightarrow 0$ - the quantum form is correct and indeed necessary.

In order to avoid unnecessary complication we first discuss the limit where we express the supercurrent in a simple form and treat the quasiparticles as in the normal state [Eqs. (48) and (49)]. In this case the least-action principle (50) yields

$$
C \frac{\hbar \ddot{\phi}}{2 e}+\frac{1}{R_{N}} \frac{\hbar \dot{\phi}}{2 e}-I+I_{c} \sin \phi=\xi_{1}(t) \cos \frac{\phi}{2}+\xi_{2}(t) \sin \frac{\phi}{2} .
$$

The right-hand side reflects the current noise $I_{N}(t, \phi)$ of the tunnel junction. It is quantum shot noise. It appears as a sum of two independent Gaussian noise terms $\xi_{1}$ and $\xi_{2}$, coupling to $\cos \phi / 2$ and $\sin \phi / 2$. Their correlation functions are

$$
\left\langle\xi_{i} \xi_{j}\right\rangle_{\omega}=\delta_{i j} 2 e^{2} \alpha_{R}^{N}(\omega)=\delta_{i j} \frac{1}{R_{N}} \hbar \omega \operatorname{coth} \frac{\hbar \omega}{2 k T}
$$

In contrast the ordinary quantum Langevin equation, for example, of Schmid, which models the Johnson-Nyquist noise of a resistor, contains only one such noise term. The noise term in (51) actually emerged in this form from our formal analysis. At the same time, this form is conceptually the simplest and most accessible, for example, for a numerical analysis. In contrast the correlation function of the total noise current depends on the actual state of the system: If the phase takes the values $\phi$ and $\phi^{\prime}$ at times $t$ and $t^{\prime}$, respectively, then

$$
\left\langle I_{N}(t, \phi) I_{N}\left(t^{\prime}, \phi^{\prime}\right)\right\rangle=2 e^{2} \alpha_{R}^{N}\left(t-t^{\prime}\right) \cos \frac{\phi-\phi^{\prime}}{2} .
$$

In the special case where the junction is operated with a constant voltage bias, such that $\phi-\phi^{\prime}=2 e V\left(t-t^{\prime}\right) / \hbar$ the power spectrum takes the familiar form obtained by Dahm et al: $:^{32}$

$$
\left\langle I_{N} I_{N}\right\rangle_{\omega}=\frac{1}{2 R_{N}} \sum_{ \pm}(\hbar \omega \pm e V) \operatorname{coth} \frac{\hbar \omega \pm e V}{2 k T} .
$$

For voltages $e V$ exceeding $k T$ and $h \omega$ the fluctuations in the tunnel current are proportional to the current itself. This is a characteristic feature of shot noise. The power spectrum is larger than the Johnson-Nyquist spectrum of an equivalent resistor. Notice that it is the factor $\cos \left(\phi-\phi^{\prime}\right) / 2$ in Eq. (53), leading to the shift by $\pm e V$ in (54), combined with the fact that $\alpha_{R}\left(t-t^{\prime}\right)$ is of the quantum form which is responsible for this difference.

As a second simple example for the use of the result (53), relevant for examples in cases where the junction is used as a mixer (see Ref. 33), we consider a tunnel junction driven by an ac voltage bias at frequency $\omega_{0}$, i.e., $V=V_{0} \cos \left(\omega_{0} t\right)$. If the voltage is small $e V_{0} \ll \hbar \omega_{0}$ the current noise at small frequencies $\hbar \omega \ll k T$ averaged over a period is

$$
\begin{aligned}
\left\langle I_{n} I_{n}\right\rangle_{\omega} & =\frac{2 k T}{R_{N}}\left[1-\frac{1}{8}\left[\frac{2 e V_{0}}{\hbar \omega_{0}}\right]^{2}\right] \\
& +\frac{1}{8}\left[\frac{2 e V_{0}}{\hbar \omega_{0}}\right]^{2} \frac{\hbar \omega_{0}}{R_{N}} \operatorname{coth} \frac{\hbar \omega_{0}}{2 k T} .
\end{aligned}
$$

In the limit where the relevant time scales are slow, i.e., $\hbar \omega, e V \ll k T$, the noise reduces to classical $\delta$-correlated or white noise. In this case, the two noise sources in (51) add up and act like one classical noise term. However, if the phase evolves faster, we explore the part of the quantum power spectrum, which (for $\hbar \omega>k T$ ) increases proportional to $\omega$. It is instructive to consider the correlation function in times: $\alpha_{R}^{N}\left(t-t^{\prime}\right)$ has a large peak at $t=t^{\prime}$ [singular if $\alpha_{R}^{N}(\omega)$ did not have a high-frequency cutoff equal to $\omega_{c}$ ], becomes negative beyond (for $\left.\left|t-t^{\prime}\right| \geq 1 / \omega_{c}\right)$, reflecting an anticorrelation in the noise which in part compensates for the equal-time peak, and decays on a time scale given by $\hbar / k T$ (we assume $\left.\hbar \omega_{c} \gg k T\right)$. If the phase varies on a time scale $\hbar / k T$, the fact that the noise couples to $\cos \phi$ or $\sin \phi$ can revert the effect of the anticorrelation. This leads to the increase of the shot noise over the ordinary quantum, i.e., Johnson- 
Nyquist noise.

The general theory of the Josephson tunnel junction represented by the action (45) leads to a quantum Langevin equation of the form

$$
\begin{aligned}
& C \frac{\hbar \ddot{\phi}}{2 e}-I-4 e \int_{-\infty}^{t} d t^{\prime}\left(\alpha_{I}\left(t-t^{\prime}\right) \sin \frac{\phi-\phi^{\prime}}{2}\right. \\
&\left.-\beta_{I}\left(t-t^{\prime}\right) \sin \frac{\phi+\phi^{\prime}}{2}\right] \\
&=\xi_{1}(t) \cos (\phi / 2)+\xi_{2}(t) \sin (\phi / 2) .
\end{aligned}
$$

Here $\xi_{1}$ and $\xi_{2}$ are again independently Gaussian stochastic variables with different correlation functions as given by the general form (47). The integrals on the left yield the quasiparticle and supercurrent in their general nonlocal form as obtained by Werthamer. ${ }^{34}$ The correlation function of the total noise current, in generalization of (53), can be written as

$$
\begin{aligned}
\left\langle I_{N}(t, \phi) I_{N}\left(t^{\prime}, \phi^{\prime}\right)\right\rangle=2 e^{2}( & \alpha_{R}\left(t-t^{\prime}\right) \cos \frac{\phi-\phi^{\prime}}{2} \\
& \left.+\beta_{R}\left(t-t^{\prime}\right) \cos \frac{\phi+\phi^{\prime}}{2}\right),
\end{aligned}
$$

which exhibits its relation to the quasiparticle and quasiparticle-pair interference current. Also, the fluctuation-dissipation theorem is apparent in this form.

In cases where the Josephson junction is operated with a fixed voltage bias the tunneling current takes the familiar form ${ }^{35}$

$$
\begin{aligned}
I_{T}(V)= & I_{n}(e V / \hbar)+\left[\mathrm{P} \int \frac{d \omega}{\pi} \frac{I_{c}(\omega)}{\omega-e V / \hbar}\right] \sin \phi(t) \\
& +I_{c}(e V / \hbar) \cos \phi(t) .
\end{aligned}
$$

The $\beta$ term gives rise to the supercurrent $(\sim \sin \phi)$ but also to the $\cos \phi$ or quasiparticle-pair interference current. (It has been pointed out in Ref. 36 that bulk scattering processes may modify this interference term, which appears consistent with experiments. This is not included in our present formulation.) In the case of a constant voltage the quasiparticle part of the power spectrum (57) becomes ${ }^{32}$

$$
\left\langle I_{N} I_{N}\right\rangle_{\omega}^{\text {quasiparticle }}=\frac{e}{2} \sum_{ \pm} I_{n}(\omega \pm e V / \hbar) \operatorname{coth} \frac{\hbar \omega \pm e V}{2 k T} .
$$

For practical calculations a hybrid description between (51) and (56) is popular and qualitatively reasonable. It treats the quasiparticle $(\alpha)$ current in its general form but ignores the interference term and the supercurrent is set to $I_{S}(t)=I_{c} \sin \phi(t)$. Consistently the noise correlations do not involve $\beta_{R}$; that means $\left\langle\xi_{i}(t) \xi_{j}\left(t^{\prime}\right)\right\rangle=\delta_{i j} 2 e^{2} \alpha_{R}(t$ $\left.-t^{\prime}\right)$.

In a different limit, namely if the phase varies slowly in time but $\ddot{\phi} \neq 0$, if furthermore $T=0$ and $\Delta \neq 0$, the quasiparticle and quasiparticle-interference term are proportional to $\ddot{\phi}_{1}$, exactly consistent with the capacitance renor- malization (34). On this level (the classical current-phase relation), the capacitance renormalization was noticed before by Tucker. ${ }^{33}$

In the case where the junction is operated such that the voltage does not vanish on the average (e.g., if a current $I$ larger than $I_{c}$ is imposed), the shot-noise power spectrum even at small frequencies $\hbar \omega / k T \ll 1$ is enhanced over the thermal noise [see Eq. (54)]. Although this agrees with the classical result, we stress again that we had to retain the quantum form of the correlation function to arrive at this result. Clearly this enhanced noise current enters into the current conservation relation (51) or (56). We thus see that the quantum form of the Langevin equation is correct in a nontrivial and important limit. In this context it is interesting to note the consequence of the Josephson relation $\hbar \dot{\phi}=2 \mathrm{eV}$. Even if the voltage changes slowly on a time scale given by $\hbar / k T$, the phase may rotate fast if $e V>k T$. Whereas usually in physics we try to use the slowest variables as independent degrees of freedom, the description of a Josephson junction is simple in terms of the fast phase. On the other hand, we must sacrifice and retain the detailed structure of the correlation function.

It would be interesting to analyze the effect of shot noise in nondriven situations by evaluating Eqs. (51) or (56). It has been conjectured by Ben-Jacob et al. ${ }^{37}$ that shot noise may be overall larger than an equivalent Johnson-Nyquist noise and thus enhance transition rates. However, their analysis is based on an ansatz for the noise, which was guided by the observation that the power spectrum (54) or (59) of the driven system is enhanced, but which violates the fluctuation-dissipation theorem. Consequently, their quantitative conclusions, except for the case $I \gg I_{c}$, are incorrect.

In a recent analysis ${ }^{19,38}$ the power spectrum of fluctuations in a normal tunnel junction with capacitance was analyzed. It turns out that in a closed system the discreteness of the available states reduces the current fluctuations, actually stronger than the above-mentioned enhancement. A Langevin equation of the type (51) or (56) in a continuous variable does not account for this feature; it is more appropriate for an open system where there is a continuum of available states.

\section{Macroscopic quantum phenomena in Josephson junctions}

We now turn to the question of how dissipation by quasiparticle tunneling affects macroscopic quantum phenomena in a Josephson junction. In particular, we are interested in the effect of dissipation on the transition rate from a superconducting into a resistive state. At low temperatures this transition occurs via quantum tunneling. Caldeira and Leggett ${ }^{5,6}$ analyzed this problem in situations where the dissipation occurs in a normal shunt resistor. They used methods developed by Langer and Callan and Coleman, ${ }^{39}$ generalized to situations where the action contains nonlocal, dissipative elements. The important point is that the tunnel rate is determined by the action of a saddle-point solution (bounce) starting and ending in the metastable potential minimum (maximum, if we work in 
imaginary times, where the potential is inversed) and crossing the barrier (valley) back and forth. Caldeira and Leggett found that dissipation inhibits the tunneling. ${ }^{40,41}$

We obtain a qualitative estimate for the difference between Ohmic dissipation and dissipation by quasiparticle tunneling simply by comparing the effective action representing the two cases. The action of Caldeira and Leggett is obtained from our approximate version (32) which we consider first-if we ignore the effect of the energy gap, i.e., replace $\alpha$ by its $\Delta=0$ limit and replace $\sin \left(\phi-\phi^{\prime}\right) / 4$ by its argument. The fact that the phase enters only in the sine function in principle makes quasiparticle tunneling less effective as a damping mechanism than an Ohmic resistor. However, since the transition to the resistive state occurs mostly for $I$ close to $I_{c}$, when the distance under the barrier is only a small fraction of $2 \pi$, this effect is usually of little importance. The gap dependence of the quasiparticle tunneling $(\alpha)$, however, leads to significant changes. A typical time scale for variations of $\phi$ (as relevant for the tunneling process) is given by the characteristic frequency of the potential, i.e., the Josephson frequency $\omega_{j}=(2 e / \hbar)\left(I_{c} / C\right)^{1 / 2}$ or, in fact, if the current $I$ is close to the critical current $I_{c}$, a small fraction of $\omega_{J}$. For most experimental parameters $\omega_{J}$ is smaller than $\Delta$. Hence, the exponential decay at $T=0$ of $\alpha(\tau)$ for large $\tau$ reduces the action of the bounce and hence the effect of the dissipation below the result of Caldeira and Leggett by a factor of order $\hbar \omega_{J} / \Delta$ (or even smaller if $I$ is close to $I_{c}$ ).

In Sec. II we showed that the effect of the quasiparticle tunneling at zero temperature for $\hbar \omega_{J}<\Delta$ is to renormalize the effective capacitance. Upon closer inspection we found that corrections from the expansion in the $\beta$ term gave rise to a further phase-dependent capacitance correction. The result for $C_{\text {ren }}$ is given in (34). It is straightforward to work out quantitatively the effect of this renormalized capacitance on the tunneling rate. If the junction is biased close to $I_{c}$ the barrier is near $\phi=\pi / 2$ and the effect of the $\beta$ term is inefficient, i.e., $C_{\text {ren }}=C+\delta C$. In contrast, in a SQUID with small inductance in an external field, $\Phi_{\text {ext }}=\Phi_{0} / 2$, the barrier is near $\phi=\pi$. This makes the capacitance change most efficient, i.e., $C_{\text {ren }}=C+\frac{4}{3} \delta C$. If the tunneling path extends over larger distances in $\phi$ (of order $\pi$ ) the effective capacitance depends on the tunneling path $\phi(\tau)$ and has to be determined self-consistently.

This capacitance renormalization can be significant for the claimed parameter values in the experiments of Jackel et $a l .{ }^{41}$ For a junction of capacitance $10^{-14} \mathrm{~F}$ and critical current $I_{c}=10^{-4} \quad \mathrm{~A}$-assuming $I_{c} R_{N}=\pi \Delta / 2 e \quad$ and $\Delta / k=15 \mathrm{~K}$-the estimate (34) yields $\delta C / C$ of order $70 \%$. This will substantially reduce the tunneling rate. We realize, however, the experimental difficulties in determining accurately the capacitance, which makes it difficult to test our conclusion.

At finite temperatures, or for small gaps $\hbar \omega_{J} \geq \Delta$, or if the dissipation occurs in a normal tunnel junction, we have to find the action of the saddle-point solution of the full nonlocal action, in the same fashion as discussed, e.g., by Caldeira and Leggett in order to determine the tunnel rate. As long as there exists a gap, the effect of quasiparticle tunneling on the quantum tunneling rate of the phase will be quantitatively weaker than that of Ohmic dissipation in a resistor.

If we consider the effect of dissipation on the quantum coherence we find qualitative differences between Ohmic dissipation and quasiparticle tunneling. It has been shown by Chakravarty, ${ }^{42}$ by Bray and Moore, ${ }^{43}$ and by others thereafter ${ }^{22,44,45}$ that the Ohmic dissipation gives rise to a certain phase transition at a critical coupling strength. For weak coupling an essentially quantum-mechanical behavior, though weakened or slowly decaying, is found; for strong coupling the behavior is essentially classical. Formally, this phase transition is related to the weak power-law decay of the kernel $\alpha_{N}(\tau) \sim 1 / \tau^{2}$, which gives rise to logarithmic interactions in the related onedimensional model of classical particles. An energy gap in the excitation spectrum leads to an exponential decay of $\alpha(\tau) \sim e^{-2 \Delta \tau / \hbar}$ for long times. Hence the interaction will be short range and no phase transition occurs. In addition, even for weak damping, the influence of quasiparticle tunneling on the quantum coherence is quantitatively weaker than that of an Ohmic resistor with the same high-voltage resistance. If macroscopic quantum coherence is to be tested in an experiment, which obviously is at the limit of what can be achieved, it is important to take advantage of these differences.

\section{ACKNOWLEDGMENTS}

This material is based upon research supported in part by the National Science Foundation under Grant No. PHY-77-27084, supplemented by funds from the U.S. National Aeronautics and Space Administration. One of us (V.A.) is supported in part by the U.S. Office of Naval Research and the Guggenheim Foundation. Another of us (G.S.) acknowledges support by the "HeisenbergProgramm" of the Deutsche Forschungsgemeinschaft.
*Present address: Institut für Festkörperforschung Kernforschungsanlage, D-5170 Jülich, Federal Republic of Germany.

${ }^{\dagger}$ Permanent address: Laboratory of Atomic and Solid State Physics, Cornell University, Ithaca, NY 14853.

1D. E. McCumber, J. Appl. Phys. 39, 3113 (1968).

${ }^{2}$ Yu. M. Ivanchenko and L. A. Zilberman, Zh. Eksp. Teor. Fiz. 55, 2393 (1968) [Sov. Phys.-JETP. 28, 1272 (1969)]; V. Ambegaokar and B. I. Halperin, Phys. Rev. Lett. 22, 1364 (1969).

3V. Ambegaokar, U. Eckern, and G. Schön, Phys. Rev. Lett. 48, 1745 (1982).
${ }^{4} \mathrm{~V}$. Ambegaokar, in Proceedings of the NATO Advanced Study Institute on Percolation, Localization, and Superconductivity, Les Arcs, 1983, edited by A. M. Goldman and S. A. Wolf (Plenum, New York, 1984).

${ }^{5}$ A. O. Caldeira and A. J. Leggett, Phys. Rev. Lett. 46, 211 (1981).

${ }^{6}$ A. O. Caldeira and A. J. Leggett, Ann. Phys. (N.Y.) 149, 374 (1983).

${ }^{7}$ R. P. Feynman and F. L. Vernon, Jr., Ann. Phys. (N.Y.) 24, 118 (1963).

${ }^{8}$ A. Schmid, J. Low Temp. Phys. 49, 609 (1982). 
${ }^{9}$ J. Bardeen, L. N. Cooper, and J. R. Schrieffer, Phys. Rev. 108, 1175 (1957).

10B. D. Josephson, Phys. Lett. 1, 251 (1962); V. Ambegaokar and A. Baratoff, Phys. Rev. Lett. 10, 486 (1963); 11, 104(E) (1963).

${ }^{11}$ We choose a formulation in terms of Feynman's path integrals [see, e.g., R. P. Feynman and A. R. Hibbs, Quantum Mechanics and Path Integrals (McGraw-Hill, New York, 1965)]. A review of the techniques involved here is given by $\mathbf{H}$. Kleinert, Fortschr. Phys. 26, 565 (1978). See also Ref. 4 for a pedagogical discussion.

12D. E. Soper, Phys. Rev. D 18, 4590 (1978).

13Y. Nambu, Phys. Rev. 117, 648 (1960).

${ }^{14}$ If we allow for slow spatial variations of $\Delta$ we find a Ginzburg-Landau-type description (see, e.g., Ref. 15). From this one can work out the effect of noncritical fluctuations on thermodynamic' properties (see, e.g., Ref. 16). It should be noted that, as long as there exists a gap in the excitation spectrum, time-dependent variations of $\Delta$ depend sensitively on the quasiparticle degrees of freedom, which may be slow (see, e.g., Ref. 17). This requires a careful analysis beyond the model Hamiltonian (1). Furthermore, it has to be noted that the model Hamiltonian (1) describes a pure system, whereas in most of the real samples impurity scattering is not weak.

15J. S. Langer, Phys. Rev. 137, A553 (1964).

16P. A. Lee and M. G. Payne, Phys. Rev. Lett. 26, 1537 (1971); J. Kurkijärvi, V. Ambegaokar, and G. Eilenberger, Phys. Rev. B 5, 868 (1972).

${ }^{17}$ See, e.g., A. Schmid and G. Schön, J. Low Temp. Phys. 20, 207 (1975).

${ }^{18}$ A. I. Larkin and Yu. N. Ovchinnikov, Phys. Rev. B 28, 6281 (1983).

${ }^{19}$ E. Ben-Jacob, E. Mottola, and G. Schön, Phys. Rev. Lett. 51, 2064 (1983).

${ }^{20}$ In the Feynman-Vernon theory (Ref. 7) the coupling is contained in their "influence functional."

${ }^{21}$ A. O. Caldeira and A. J. Leggett, Physica 121A, 587 (1983); A. O. Caldeira, Ph.D. thesis, University of Sussex 1980.

${ }^{22}$ S. Chakravarty and A. J. Leggett, Phys. Rev. Lett. 52, 5 (1984).

${ }^{23}$ L. P. Kadanoff and G. Baym, Quantum Statistical Mechanics (Benjamin, New York, 1962). Alternatively, we may use Keldysh's formulation: L. V. Keldysh, Zh. Eksp. Teor. Fiz.
47, 1515 (1964) [Sov. Phys._JETP 20, 1018 (1965)]; compare, e.g., Ref. 18.

${ }^{24}$ G. Zwerger, Z. Phys. B 47, 129 (1982); A. J. Leggett, Phys. Rev. 30, 1208 (1984).

${ }^{25}$ F. Guinea, Phys. Rev. Lett. 53, 1268 (1984); L. D. Chang and S. Chakravarty, Phys. Rev. B 29, 120 (1984).

${ }^{26}$ A. Widom and T. D. Clark, Phys. Rev. Lett. 48, 63 (1982); 48, 1572 (1982); A. O. Caldeira and A. J. Leggett, ibid. 48, 1571 (1982).

${ }^{27}$ A. O. Caldeira and A. J. Leggett (unpublished).

${ }^{28}$ R. H. Koch, D. J. van Harlingen, and J. Clarke, Phys. Rev. Lett. 45, 2132 (1980).

${ }^{29}$ I. R. Senitzky, Phys. Rev. 119, 670 (1960).

30J. Kurkijärvi, Phys. Lett. 88A, 241 (1982).

${ }^{31}$ H. Metiu and G. Schön, Phys. Rev. Lett. 53, 13 (1984).

${ }^{32}$ A. J Dahm, A. Dennenstein, D. N. Langenberg, W. H. Parker, D. Rogovin, and D. J. Scalapino, Phys. Rev. Lett. 22, 1416 (1969).

33J. R. Tucker, IEEE J. Quant. Electron. QE-15, 1234 (1979).

${ }^{34}$ N. R. Werthamer, Phys. Rev. 147, 255 (1966).

${ }^{35}$ See, e.g., B. D. Josephson, in Superconductivity, edited by R. D. Parks (Dekker, New York, 1969), Vol. 1.

${ }^{36}$ A. B. Zorin, I. O. Kulik, K. K. Likharev, and J. R. Schrieffer, Fiz. Nizk. Temp. 5, 1138 (1979) [Sov. J. Low Temp. Phys. 5, 537 (1979)].

${ }^{37}$ E. Ben-Jacob, D. J. Bergman, B. J. Matkowsky, and Z. Schuss, Phys. Lett. 99A, 343 (1983).

${ }^{38}$ T. L. Ho, Phys. Rev. Lett. 51, 2060 (1983).

${ }^{39}$ J. S. Langer, Ann. Phys. (N.Y.) 41, 108 (1967); C. G. Callan and S. Coleman, Phys. Rev. D 16, 1762 (1977).

${ }^{40}$ This conclusion was qualitatively confirmed in the experiments of R. F. Voss and R. A. Webb, Phys. Rev. Lett. 47, 265 (1981).

${ }^{41}$ See also R. de Bruyn Ouboter and D. Bol, Physica 112B, 15 (1982); L. D. Jackel, J. P. Gordon, E. L. Hu, R. E. Howard, L. A. Fetter, D. M. Tennant, R. W. Epworth, and J. Kurkijärvi, Phys. Rev. Lett. 47, 697 (1981).

${ }^{42}$ S. Chakravarty, Phys. Rev. Lett. 49, 681 (1982).

${ }^{43}$ A. J. Bray and M. A. Moore, Phys. Rev. Lett. 49, 1545 (1982).

${ }^{44}$ A. Schmid, Phys. Rev. Lett. 51, 1506 (1983).

${ }^{45} \mathrm{~V}$. Hakim, A. Muramatsu, and F. Guinea, Institute for Theoretical Physics (Santa Barbara) Report No. NSF-ITP84-02 (unpublished). 